\section{IDDF2019-ABS-0262 THE INFLUENCE OF EXTENT OF LYMPH NODE DISSECTION ON THE SURVIVAL OF INTRAHEPATIC CHOLANGIOCARCINOMA WITH LYMPH NODE METASTASIS}

Ying Zhang ${ }^{*}$, Hongpeng Chu, Shuling Chen, Hong Peng, Sui Peng, Ming Kuang. The First Affiliated Hospital of Sun Yat-sen University, Guangzhou, China

\subsection{6/gutjnl-2019-IDDFabstracts.299}

Background Surgical resection remains the only curative therapy for intrahepatic cholangiocarcinoma (ICC). Lymph metastasis is one of the major risks for ICC patients with surgery. The benefit of lymph node dissection is controversial. The aim of this study was to investigate the extent of lymph node dissection on the survival of ICC.

Methods From June 1998 to June 2018, a consecutive 78 patients with primary ICC underwent resection with negative surgical margin (R0) and pathological lymph node metastasis $(\mathrm{LN}+)$, were enrolled in our study. Patients were divided into the lymph node sampling group (A) and the regional dissection group (B) according to the extent of lymph node dissection. Multivariate COX regression analysis was performed to identify the prognostic factors for the overall survival (OS) and the recurrence-free survival (RFS). OS, RFS, costs and postoperative complications were compared between the two groups. We also investigated number, proportion and groups of $\mathrm{LN}+$ in group $\mathrm{B}$. Results Median OS was 8.33 months for group A $(n=25)$ and 12.79 months for group B $(n=53, P=0.112)$. The extent of lymph node dissection was not an independent prognostic factor for OS $(\mathrm{HR}=0.68,95 \% \mathrm{CI}=0.38-1.19, P=0.177)$. The corresponding RFS were 3.24 months and 5.95 months for group A and B. RFS of group B was significantly longer than that of group A $(P=0.003)$. The extent of lymph node dissection $(\mathrm{HR}=0.47,95 \% \mathrm{CI}=0.22-0.97, P=0.041)$ and tumor differentiation $(\mathrm{HR}=2.01,95 \% \mathrm{CI}=1.08-4.05, P=0.028)$ were independent prognostic factors for RFS. In group B, the number of $\mathrm{LN}+(>5)$ significantly influenced RFS $(\mathrm{HR}=15.01$, $95 \% \mathrm{CI}=2.63-85.52, \mathrm{P}=0.002)$. However, postoperative complications $(20.0 \%$ vs $56.6 \%, \mathrm{P}=0.003)$ were more frequent in group B than in group A. No significant difference was found in postoperative hospital stay and cost.
Conclusions For LN+ patients, RFS could be significantly prolonged by regional dissection. However, regional dissection did not improve the long-term OS and might lead to higher rates of complications.

\section{IDDF2019-ABS-0271 LIVER STIFFNESS MEASUREMENT IN NON- ALCOHOLIC FATTY LIVER DISEASE: TWO IS BETTER THAN ONE}

${ }^{1}$ Kee Huat Chuah*, 'Lee Lee Lai, ${ }^{2}$ Nik Raihan Nik Mustapha, 'Sanjiv Mahadeva, 'Wah Kheong Chan. 'Gastroenterology and Hepatology Unit, Department of Medicine, Faculty of Medicine, University of Malaya, Kuala Lumpur, Malaysia; ${ }^{2}$ Department of Pathology, Hospital Sultanah Bahiyah, Alor Setar, Kedah, Malaysia

\subsection{6/gutjnl-2019-IDDFabstracts.300}

Background Repeating liver stiffness measurement (LSM) was recently reported to improve diagnostic accuracy in patients with non-alcoholic fatty liver disease (NAFLD). However, there are to date no other studies confirming this finding.

Aim To evaluate the accuracy of repeating LSM for the diagnosis of NAFLD with high liver stiffness measurement.

Methods Adult NAFLD patients who had two FibroScan within 6 months prior to liver biopsy in a university hospital were included. F3-F4 fibrosis was excluded using LSM cut-off of $7.9 \mathrm{kPa}$.

Results A total of 140 patients were recruited. 85\% (119/140) patients had high LSM $(\geq 7.9 \mathrm{kPa})$ on baseline scan. Among them, 27\% (32/119) had low LSM $(<7.9 \mathrm{kPa})$ on the repeat scan, with only $6 \%(2 / 32)$ having F3 fibrosis. $47 \%(41 / 87)$ patients with both high LSM had F3-F4 fibrosis. All 21 patients with low LSM on baseline scan had F0-F2 fibrosis, except one patient with high repeat LSM who had F3 fibrosis (figure 1). Repeating LSM strategy, where only patients with high LSM on both scans are considered for liver biopsy, will be able to reduce the number of patients being considered for liver biopsy need from $85 \%$ to $62 \%$. Sensitivity, specificity, positive predictive value, negative predictive value based on only the baseline scan was 98\%, 21\%, 36\% and 95\%, while based on repeat LSM strategy was 93\%, 52\%, 47\% and 94\% respectively.

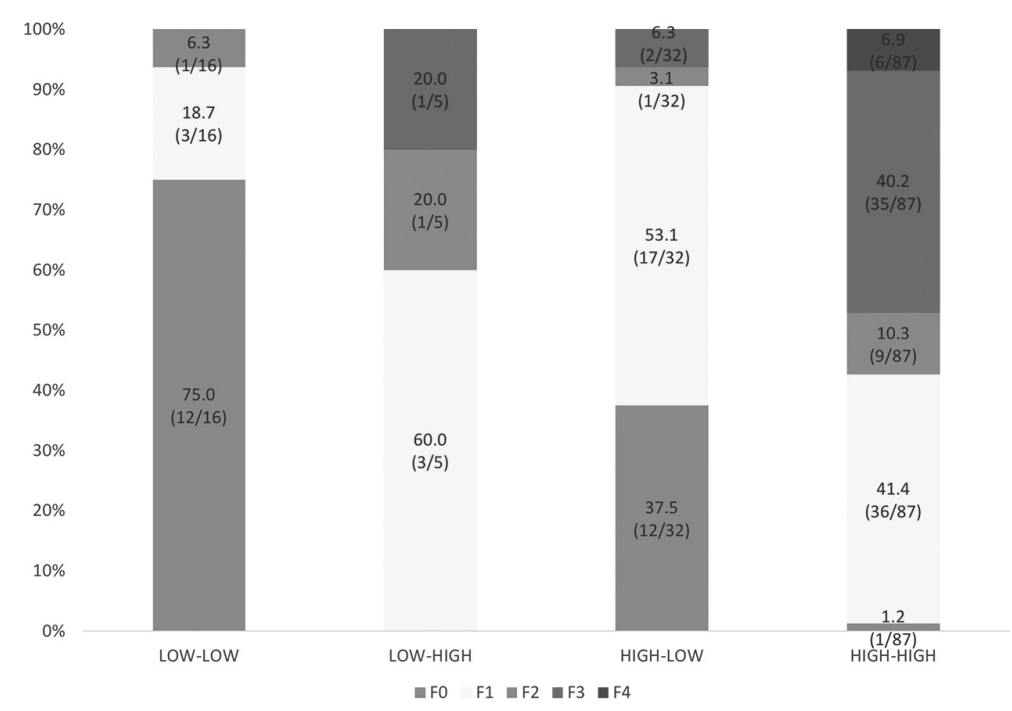

Abstract IDDF2019-ABS-0271 Figure 1 Percentage of each of the histological groups based on repeated LSM examinations categories low LSM 7-9kPa high LSM 7-9 